\title{
A NEW TENTATIVE THEORY OF VISUAL SPACE PERCEPTION
}

\author{
TOKUMICHI NISHI
}

Shuko College, Ichinoscki, Iwate Prefecture

I

Since the writer started the study of visual space perception about thirty years ago, he investigated extensively various theories or laws that account for every phenomenon of visual space, and found out that a theory adopted by an author to explain a certain phenomenon is restricted to that one alone but is inapplicable to another phenomenon. For instance, the explanation of the lighthouse phenomenon by Bernstein or that of the phenomenon in the high Rockies may solely be applicable to the respective phenomenon.

The writer thinks that a theory of the visual space as a theory must be applicable to various phenomena concerning the visual space perception.

The writer has made researches on the visual space and found a special principle that can explain integrally every phenomenon pertaining to the three dimensional field.

Through various observations the writer has perceived a certain factor that plays a very important part in the visual space. Objects which lie beyond a certain 'limit' from 0 are generally localized at the very 'limit'. He calls it the 'limit of the depth perception'.

On the ground of this 'depth limit', e.g. the stars are all localized at the equal distance From 0 . Therefore the starry sky must look as a vault. The clouds should be an 'even roof', but the clouded sky appears as a vault also, because the part of the clouds farther than the 'depth limit' will be localized at the 'depth limit'. The blue sky will fall under the same category. The innumerable particles in the atmosphere which reflect rays of shorter wave-lengths looking like a blue surface may be localized, very obscure as it is, at the 'depth limit'.

But the 'vault' appears generally somewhat flat. The reason for it may be as follows: The 'depth limit' is relatively of small size, and so the continuous surface of the terrain can prolong our discerning range, conspicuously in the horizontal direction. Thereby we get to the 'secondary depth limit'.

Thus the 'moon illusion' occurs, as the distances of the localization in both directions (vertical and horizontal) differ considerably. It should be noticed that the form of the sky does not condition the 'moon illusion', but that both the form of the sky and the apparent sizes of the moon should be determined by one and the same factor, the 'depth limit'.

As this 'limit' lies at comparatively short distance from 0 , the surface of the earth must look to be a concave vault, if it is observed from very high altitude, as illustrated by Hann et al.

The phenomenon in the high Rockies may be explained also by the same theory. As distant, large mountains are localized at the 'depth limit' (the secondary one) which is not so far, they must look as near, small ones. What is more, the fresh green color heightens the effect. 
The phenomenon in the high Rockies may be explained also by the same theory. As distant, large mountains are laocalized at the 'depth limit' (the secondary one) which is not so far, they must look as near, small ones. What is more, the fresh green color heightens the effects.

The lighthouse phenomenon-when observed at the foot of a lighthouse, the beam of light from it assumes the form of a sky-dome-is no exception to the rule, i.e. the beam beyond the 'depth limit' may be localized at the very 'limit'.

It has been ascertained that the moon near the horizon appears larger when observed in a bright moonlit night, but the sun near the horizon appears larger when it is covered with veil-like clouds. In the former case, the moon is observed across the stretches of spatial continuum of the ground between it and 0 , so it must seem larger, it being localized at the 'secondary limit'. In the latter, the sun is observed on the same condition as the former. On the contrary, when the sun is observed in a cloudless sky, it can be observed only for an instant so or through a smoked glass, so that 0 cannot glance over the stretches of the spatial continuum, it must appear smaller, it being localized at the 'primary depth limit'.

It seems a matter of astronomers' common knowledge that the starry sky looks hemispheric (not flattened). The reason for it must be the lack of the continuous surface of the terrain. A fire at night being perceived nearer can be explained similarly.

The above is the gist of "A New Tentative Theory of Visual Space Perception (I)" $\star$

The following is the summary of Part II. ${ }^{\star}$ In it the writer has aimed at clearing up the cause of that cardinal mental process, the 'depth limit'. For the first, he has scrutinized the relationship between a retinal image and a percept.

II

In general, a percept is confounded with a stimulus as exemplified by Koff ka's 'distant stimulus' which means' a stimulus for our perception of a table'. Now what is the relation between the 'proximal stmuli' and the 'distant stimulus'? According to Koff ka: 'The distant stimulus', the table in the geographical environment, is a stimulus for our perception of a table; the 'proximal stimuli', the excitations to which the light rays coming from the table give rise are the stimuli for our perception. The distinction between 'the perception of a table' and 'the perception' is very ambiguous. Presumably Koffka has meant 'the perception' as the inducement to neural excitations. If that is the case, 'the perception of an object' entails 'the distant stimulus' and 'the proximal stimuli'. So his theory, in a sense, is dualistic. Perhaps all others' theories may be similar.

The writer's own theory is monistic. The point follows: The cone of light waves which is radiated or reflected from a physical existence enters the eye and is focused as an image in the retina and through the highly complicated psychophysical mechanism, visual parts of the brain as the central agent, the 'physical force' is transmuted into a 'psychological one', a special energy, and finally the latter is projected outward producing the percept.

It must be noticed that the physical existence itself cannot be directly perceived. It is only the source of stimuli.

* Annual Report of the Gakugei Faculty of the Iwate University, 1957, 11, Pt. I, 1-17.

$\star \star$ Tohoku Psychological Folia, 1958, 17, Fasc. 1, 1-20. 
Thus the writer does assume that the 'visual mechanism' must have the power to project the specific energy according to various conditions. These conditions may be classified into two groups. The one is the main factor and the other the secondary one. The former chiefly consists of distance-variables or the lengths from 0 to the physical existence.

Within a very short range of 0 , the 'projecting' operation is so exact that each 'projecting' point of the retinal image coincides with each reference point of the physical existence. So the mathematicalformulae, particularly those of geometry, may be applied rather rigorously.

In proportion as the real physical distance increases, however, the 'projected' position, of the retinal image decreases representing a serial reduction of depth localization, so that the 'depth limit' at length turns up.

The secondary factor consists of several cues which reinforce the main factor. Among others the most important one is the continuity of the surface.

The size of the retinal image or the visual angle of the same object may be diverse markedly in reference to distance as above alluded to, the perception of that object nevertheless may be almost equal. That must be the essential quality of the 'size constancy'. The nearer the projected position from 0 is fixed, the larger the range of the size constancy (i.e. the volume of uniform size, so to speak, throughout its entire length) becomes. According to the writer's experiment, this trend was outstanding at the distances less than $5 \mathrm{~m}$. from 0 .

It is expressed in a co-ordination. The abscissa gives the distance from 0 to the standard stimulus, and the ordinate the percentage of the decrease of the area. The curve of 'size constancy' falls precipitously at the distances less than $5 \mathrm{~m}$. from 0 , and then lowers gradually. This characteristic must be the 'law of size constancy' in the strict sense of the word.

The writer conducted another experiment. The distances used were ranged from $50 \mathrm{~m}$. to $500 \mathrm{~m}$. He obtained the result that an object kept on appearing as the same in size until about $8 \%$ of the area either increased or decreased at cach observed point. That is nothing but Weber's law.

The phenomenal facts called 'size constancy' have, in reality, several aspects, though the term has been supposed to have only one import. He can ennumerate three senses. Two of them have been already referred to. The writer must mention one more aspect of constancy which is to obey 'the law of visual angle'. Two (or more) objects lying farther than the 'depth limit' appear equal in size if their visual angles or the retinal images are similar. For instance, the sun and the moon appear almost the same in size. That is the case in point.

That the visual organ is no other than a 'projecting mechanism' may be proved fully by several phenomenal facts. One of these to verify for certain the 'psychological projection theory' is a phenomenon referred to as Emmert's law. When an after-image is projected outward, it is generally said, its localization is indefmite with respect to size and distance*.

The process of projecting an after-inage belongs to the same category of perception itself. The only difference consists in that which the former has the process of excitations or the image alone on the retina lacking, at the very moment of projecting those excitations, the reference physical existence which has caused those excitations. Oin the contrary, a perception

* On this subject, the writer conducted experiments and has got a certain essential result. He will publish it opportunely. 
has the physical existence and takes aim at it and projects the special 'psychical energy.'

The process of the 'projecting' energy may be verified fully also by photographs taken at the very close range. The foremost part of the picture of a lengthwise long object comes out extremely magnified, and then it tapers off gradually.

This disproportion of the picture arises out of the physical agency which will project light rays reflected from every point of the original on the film or the plate of the camera according to the physical laws of light rays. The form of the retinal image may be quite the same. The reason why the form of the percept is not disproportional must be as follows: When we seen an object or original within a very short range, the most magnified part of the retinal image will be projected at the foremost part of the object, and the more the form of the part of the retinal image tapers, the more the projected points are localized farther and at length get to theterminal. So the form of the percept unlike the photograph is well proportioned. Anyone will find out that a photograph taken at a relatively short range, e.g. thatof a room, a garden, or swimming pool appears far beyond the original.

The reason may be: In a photo, as before stated, the foremost part comes out most magnified, the other tapers away gradually. But on a natural observation, a scene similar to the very picture of the photo having such a proportion of the foremost part to the last is really a very large scene as a matter of course. So we would turn the picture of a photo into a directly seen view and would become possessed with an illusion to substitute the former for the latter. In general, unlike animate things, inanimate objects become grandeur instead of being deformed through the partial magnification.

On the other hand, in a photograph of a distant, high mountain the depth from 0 is compressed such an extent that when the photo is turned into a directly 'seen view, the mountain may be localized, in general, remarkably ahead of of the 'depth limit' owing to the lack of the continuum of the terrain, so that a distant, high mountain in a photo appears not so high as that seen directly. It resembles the phenomenon in the high Rockies. It is a matter of course that we observe a mountain at the 'depth limit' (the secondary one).

The writer's theory which may be called inclusively a special 'projection theory' including the 'depth limit' may explain the miscellaneous phenomenon reasonably, systematically, and consistently.

MS. received I 10,60

Tokumichi Nishi (西 德 道 1886-) Graduated from Tokyo Univ., in 1915. 1925-43, Prof. of Hirosaki National College; 1944-51, Lecturer at the Training School for Meteorological Technicians attached to the Central Meteorological Abservatory; 1951- Prof. of Iwate Univ. Retired under the age limit in 1958; 1958Prof. of Shuko College.

P. S. The writer has a good many offprints of his theses. If you wants a copy of part I, or part II, or both please apply to the following address. the writer will send you either or both in order of receipt:

Prof. T. Nishi, Shuko College, Ichinoseki, lwate, Japan. 